RESEARCH ARTICLE

\title{
An Adaptive Trust Model Based on Fuzzy Logic
}

\author{
Um Modelo de Confiança Adaptativo Baseado em Lógica Fuzzy
}

\author{
Jeferson José Baqueta1*, Mariela Morveli-Espinoza1, Gustavo A. Giménez-Lugo ${ }^{1}$, Cesar A. \\ Tacla $^{1 *}$
}

\begin{abstract}
In cooperative environments is common that agents delegate tasks to each other to achieve their goals since an agent may not have the capabilities or resources to achieve its objectives alone. However, to select good partners, the agent needs to deal with information about the abilities, experience, and goals of their partners. In this situation, the lack or inaccuracy of information may affect the agent's judgment about a given partner; and hence, increases the risk to rely on an untrustworthy agent. Therefore, in this work, we present a trust model that combines different pieces of information, such as social image, reputation, and references to produce more precise information about the characteristics and abilities of agents. An important aspect of our trust model is that it can be easily configured to deal with different evaluation criteria. For instance, as presented in our experiments, the agents are able to select their partners by availability instead of the expertise level. Besides, the model allows the agents to decide when their own opinions about a partner are more relevant than the opinions received from third parties, and vice-versa. Such flexibility can be explored in dynamic scenarios, where the environment and the behavior of the agents might change constantly.

Keywords: Trust Model - Reputation — Social image - Partner Selection
\end{abstract}

Resumo: Em ambientes cooperativos é comum que os agentes delegem tarefas uns aos outros para atingir seus objetivos, uma vez que um agente pode não ter as capacidades ou recursos para atingir seus objetivos sozinho. Contudo, para selecionar bons parceiros, o agente precisa lidar com informações sobre as habilidades, experiências e objetivos desses parceiros. Nessa situação, a falta ou imprecisão de informações pode afetar o julgamento do agente sobre um determinado parceiro, e consequentemente aumentar os riscos de delegar um objetivo a um agente não confiável. Portanto, neste trabalho, apresentamos um modelo de confiança que combina diferentes tipos de informação, como imagem social, reputação e referências para produzir uma informação mais precisa sobre as características e habilidades dos agentes. Um aspecto importante de nosso modelo de confiança é que ele pode ser facilmente configurado para lidar com diferentes critérios de avaliação. Por exemplo, como apresentado em nossos experimentos, os agentes são capazes de selecionar seus parceiros por disponibilidade ao invés do nível de maestria. Além disso, o modelo permite que os agentes decidam quando suas próprias opiniões sobre um parceiro são mais relevantes do que as opiniões recebidas de terceiros, e vice-versa. Essa flexibilidade pode ser explorada em cenários dinâmicos, onde tanto o ambiente como o comportamento dos agentes podem mudar constantemente.

Palavras-Chave: Modelo de Confiança - Reputação - Imagem social - Seleção de Parceiros

${ }_{1}^{1}$ Programa de Pós-Graduação em Engenharia Elétrica e Informática Industrial, Universidade Tecnológica Federal do Paraná (UTFPR), Curitiba - Paraná, Brazil

*Corresponding author: jefersonbaqueta@gmail.com, morveli.espinoza@gmail.com, gustavogl@utfpr.edu.br, tacla@utfpr.edu.br DOI: http://dx.doi.org/10.22456/2175-2745.110818 • Received: 20/01/2021 • Accepted: 29/08/2021

CC BY-NC-ND 4.0 - This work is licensed under a Creative Commons Attribution-NonCommercial-NoDerivatives 4.0 International License.

\section{Introduction}

In Multi-agent systems (MAS), trust and reputation models have been adopted as an important solution to ensure security and efficiency [1][2]. These models offer mechanisms to penalize inappropriate behaviors of agents and improve the partner selection process in uncertain situations [3]. Generally, in these kinds of models, agents are rated (e.g., good or bad) as they interact with each other. Thus, a good partner may be identified based on the ratings about its behavior, which are performed along the time considering a certain context (i.e., a set of propositions describing a state of the world) [4].

As discussed in [4], when an agent decides to delegate a goal to a partner, this agent puts itself in a risk position since delegation involves relying on someone. Thus, it is 
important that before delegating a task, the agents consider all possible available information to make the best decision. In the literature, there are several works that use trust to solve the delegation problem among agents (e.g., [5] [6] [7]). However, in many cases, the trust concept is modeled based on just the agents' capabilities or the task requirements (e.g., cost, time, and quality), that is, on the internal factors. As pointed out by [4], such a simplified trust modeling may not be adequate for scenarios affected by constant changes.

From the point of view of the dynamic studies of trust, the basic beliefs that an agent has about its partner, and vice-versa, may change during the interaction between them [4]. For example, due to some external factor (e.g., like opportunities, obstacles, adversities, and interference), the partner's abilities might be changed, consequently affecting the dependence relationships between it and the other agents. For instance, consider the case of a violinist [4], even trusting him for playing very well (i.e., assuming that his abilities to play the violin are extraordinary), suppose he has to do the concert in an open environment and the weather conditions are particularly bad, it is very cold (adversities). Such conditions may modify the specific hand abilities of the violinist and his performance (obstacles). Moreover, it is possible that a special distracted, inattentive, noisy audience could modify his willingness and consequently again his performance (interference).

Therefore, in a dynamic scenario, a more sophisticated trust model that supports the internal and external factors may ensure better success chances regarding the execution of tasks. An example of this kind of approach is presented in [8], where the authors propose a trust model that combines the internal and external factors into a trust measure. This model is adopted in [4] to evaluate the risks associates with the delegating task process.

In this work, we present a trust model that combines different pieces of information, such as social image, reputation, and references to produce more precise information about the characteristics and abilities of agents (by cross-correlating several perspectives). Even though our trust model does not directly deal with external factors, the decision of trusting in someone is made based on the context of the agents (i.e., information that change over time as the agents interact with one another). In this sense, our trust model can be employed for the partner selection process, even in dynamic scenarios, where the behavior of agents may change over time. Additionally, from the use of a fuzzy classifier, our trust model can be dynamically configured according to each agent's world state, which allows the model to adapt itself to different needs and demands of agents that may occur due to changes in context. In particular, the trust model presented herein is based on the model proposed in [8]. The following points summarise the main differences between our approach and the original one:

- Information sources: in [8], it is assumed that the inputs of the trust model come from beliefs produced through direct experiences of agents. In our approach, the trust measure is computed from the social image (agent's opinion) [3], reputation (third party opinions) [9], references (recommendations about services performed by agents in the past) [1], and the availability of agents. The adoption of multiple sources of information increases the volume of the available information in the system. In this case, the agents can make their trust decision by combining a variety of alternative sources of information, which allows the agents to have a more precise trust measure (by cross-correlating several perspectives) and to cope with situations where some of the sources may not be available [10]. Besides, the use of different sources of information allows the agents to estimate a trust measure about a partner based on the information shared with them by other agents [3].

- Fuzzy classifier: in the model presented in [8], some parameters, such as the causality relationships among the concepts of the model, have their values predefined and need to be configured by a human specialist. In our approach, this configuration is performed dynamically since these causality relations are defined through a fuzzy classifier that operates on a rule base built from variables associated with the context of agents.

The rest of this paper is organized as follows. Section II presents some basic concepts and definitions adopted in this work. Section III presents the proposed trust model and discusses its structure and the modeling details considered during the development of the model. Section IV discusses the implementations and the case study used in our experiments. Section V describes the experimental results and discusses the result obtained. The conclusions and future work are summarized in Section VI. At last, in Section VII some related works are presented and discussed.

\section{Basic Concepts}

This section reviews some of the main definitions and concepts regarding the social control of agents adopted in this work. As discussed in [11], in a social control approach, agents themselves are capable of penalizing non-desirable behaviors (e.g., not selecting a given partner for a task). Herein, we discuss trust and reputation mechanisms, two examples of this kind of control.

\subsection{Trust}

In cooperative environments, it is usual that agents delegate tasks to each other to achieve their goals. In many cases, an agent may not have the capabilities or resources to achieve its goals alone. In this kind of situation, the agents need to deal with information about the abilities, experiences, and goals of possible partners. As discussed in [4] [12], the act of trusting someone is not a simple action because it requires the fulfillment of some conditions. Firstly, the agent must perform a preventive evaluation about the characteristics and virtues of all possible partners (i.e., validating the minimum 
requirements for trusting in them). Following, the agent compares the potential candidates, considering the risks and costs of delegating the task. In the last stage, the agent must select a partner and delegate the task to it (i.e., establishing a trust relationship with the selected partner).

As discussed in [13], trust is a multidisciplinary concept, which has been used in different disciplines to model different types of relationships. Therefore, in this work, we adopt the trust definition suggested by [14], where trust is defined through five components, which are represented by the tuple $\operatorname{TRUST}\left(X, Y, C, \tau, G_{x}\right)$. This tuple can be read as, $X$ trusts $Y$ in a context $C$ for performing an action $\alpha$ (through the task $\tau$ ) and obtaining as result $p$ (the outcome expected by $X$, which corresponds to $X$ 's goal $\left(G_{x}=g_{x}\right)$. Consequently, when an agent delegates a task to a partner, there is a trust relationship between them, in which the trustor agent creates expectations about the fulfillment of the task, and hence, starts relying on its partner.

\subsection{Reputation}

The reputation concept can be seen as a mechanism to penalize inappropriate behaviors of agents and improve the partner selection process [15]. Thus, reputation has been associated with the opinion that others have about someone [3]. Moreover, as defined in [2], reputation can be understood as a meta-belief, since it is produced based on third party opinions that circulate in some society. Hence, reputation can be defined as a general opinion shared by the majority of the members of society about something or someone.

In general, an agent can compute the reputation of a target by aggregating the impressions of other agents about it. Therefore, an impression can be defined as a rating about the behavior of an agent considering certain evaluation criteria. In literature there are many ways to compute such a value [9], [10], [2] [16]. In this work, we use the weighted mean of the impressions. This approach groups a set of impressions together to form a single summary value, giving more relevance to impressions received most recently. The equation used to compute the reputation is the following:

$$
R^{t}\left(I B D_{f}^{x}\right)=\sum_{t_{i} \in I B D_{f}^{x}} p\left(t, t_{i}\right) \cdot W_{i}
$$

where $R^{t}$, represents the reputation value in time $t, I B D_{f}^{x}$ represents all the impressions from the belief base of agent $x$ filtered by one or more criteria, which are selected according to a given filter $f$. For instance, a filter could be used to select all impressions about a taxi driver agent, where the attribute safety driving is good or higher. In addition, $p\left(t, t_{i}\right)$ represents a time dependent function that prioritizes impressions produced in a certain time $t_{i}$ closer to current time $t$, and $W_{i}$ indicates a value, within interval $[0,1]$, assigned to an evaluating criterion. A complete discussion and specific details about this equation can be found in [9].

As specified in [9], we also calculate the reliability of reputation $\left(R^{t}\right)$. Such a value establishes how much trustworthy the reputation of someone is. Basically, the reliability value is computed taking into account two factors: $(i)$ the number of impressions adopted to calculate the reputation, since isolated experience is not enough to make a correct judgment of someone; and (ii) subjective reputation deviation, which aims at measuring the variability of impressions used in the reputation computing. According to [9], high variability indicates a low credibility reputation.

\subsection{Social Image}

Social image is a belief usually produced from the direct experiences of the agent, and hence, expressing a more personal opinion about a target agent [3]. Additionally, as discussed in [2], the social image is a social evaluation regarding the functions or roles that a target agent plays. Furthermore, different members of a society may have different images of the same target. For instance, in a commerce scenario, agent $X$ may have the social image that the prices of books sold by agent $Y$ are fair (agent $X$ has a good social image of agent $Y$ as a book seller). On the other hand, at the same time, agent $X$ may believe that the magazines sold by agent $Y$ are very expensive (agent $X$ has a bad social image of agent $Y$ as a magazine seller). Therefore, for agent $X$, agent $Y$ is seen from different perspectives, according to the type of product that agent $X$ wants to buy.

As the social image of an agent can be computed from an aggregation of impressions, we also use the Equation 1 to compute the social image of agents. However, in this case, the social image value is calculated only using the own impressions of the agent, which are obtained from direct interactions with a target.

\subsection{Social Evaluation: Social Image and Reputation}

As the social image is produced based on the direct interaction between the agents, this type of social evaluation tends to express the real aspects of the behavior of a partner [3]. On the other hand, the reputation does not bind the speaker to commit himself to the truth value of the evaluation communicated but only to the existence of rumors about it [15]. Therefore, in the case of reputation, unlike sincere communication (i.e., when the social image of an agent is shared with others), only the acceptance of a meta-belief is required in the communication. In this sense, as discussed in [11], according with the situation, one type of social evaluation may be more relevant than another for the agent. For example, in a society where the agents can lie about the behaviors of their partners, in order to guard against liars, an agent can decide only trusts its own direct experiences (i.e., using the social image to take decisions). On the other hand, in a situation where the agent needs to interact with a partner, but it does not have any personal evaluation about such a partner, the partner's reputation that circulates in the society can be used by the agent to decide whether and how to interact with its partner. 


\subsection{Certified Reputation}

As discussed previously, when an agent $a$ does not have sufficient knowledge about the behavior of another agent $b$, the $b$ 's reputation can be exploited to estimate the trustworthiness of $b$. However, as reputation does not require that the agents commit themselves to the truth of the communicated information, $a$ cannot verify the veracity of the $b$ 's reputation. Such a characteristic decreases the reputation's immunity to malicious agent behavior [1] [17]. Malicious agents are able to share fake information with other agents, making different types of attacks, as self-promotion and slandering attacks. In a self-promotion attack, a malicious agent improves its own reputation by spreading fake positive impressions about itself. On the other hand, in a slandering attack, a malicious agent uses negative fake impressions to decrease the reputation of other agents.

In the certified reputation, the information (impressions) shared by agents are evaluations presented by a partner (agent $b$ ) about itself, which have been obtained from other agents in past interactions [10]. Thus, $b$ can provide its certified impressions to any agent that wishes to interact with it in the future, similar to job references. Moreover, in this case, the reputation of an agent can be compared to its references in order to check inconsistencies between them. Furthermore, each certified impression (reference) is signed by the appraiser agent to a partner and then validated by a certifying agent through an encryption mechanism to ensure reference's credibility [18]. This process prevents self-promotion and slandering attacks since all references must be certified before being shared with other agents.

In this work, we consider that a partner can store its references and share them with other agents. In our case study, all agents are considered honest (i.e., they are not able to perform self-promotion and slandering attacks). Besides, we are not employing an encryption mechanism to certify the references of agents. In our case, the references are used as an extra source of information to estimate the trustworthiness of a partner, similar to the social image and reputation. To compute the references of the agents, we are using the equation 1 , since this process consists of aggregating the references received by an appraiser agent.

\section{The Proposed Trust Model}

In this section, we present our trust model. Herein, the structure of the model and the concepts adopted to compound the trust of agents are discussed and explored. We also present design decisions taken for the elaboration of the model.

\subsection{Fuzzy Cognitive Map (FCM)}

As shown in Figure 1, our trust model uses a Fuzzy Cognitive Map (FCM) [19]. An FCM is a set of nodes connected by weighted edges, where the nodes represent concepts that describe the evaluation characteristics, and the weighted edges represent the causal relationships that exist among the concepts [20]. In part, the FCM structure shown in Figure 1 is inherited from the model proposed [8]. However, in our case, the trust measure is computed based on a different set of components (i.e., social image, reputation, know-how, and availability).

As discussed in [4], besides the simplicity of implementation, FCMs can represent the trust components and combine them into a trust measure through a process of propagation of values. Moreover, being based on Fuzzy Logic, it is possible to use natural language labels to represent a specific real situation. Nevertheless, it is important to remark that our trust model does not depend on the FCM structure. Basically, any other structure that allows combining the different trust components could be used in the place of the FCM.

Additionally, in our approach, the FCM is built to express the reasoning sequence adopted by an agent to decide whether trust or not in another agent. In this sense, firstly, in order to produce a general evaluation concerning the partner's behavior, the agent compares its own opinion (social image) to third parties opinions (reputation). Next, the agent's opinion is compared to partner's know-how (references). Finally, the agent decides whether the availability of the partners is more important than the partner's abilities.

In order to compute a trust measure, the values of the input nodes of the FCM are propagated until the output node. This process is performed through equation 2 , which takes into account the influence of the interconnected concepts:

$$
V_{j}^{t}=f\left(\sum_{i=1 \wedge i \neq j}^{k} V_{i}^{t-1} \cdot W_{i j}\right)
$$

where $V_{j}^{t}$ represents the value of the concept $C_{j}$ at time $t$, $V_{i}^{t-1}$ represents the value of the concept $C_{i}$ at time $t-1, W_{i j}$ represents the weight of the edge that connects the concepts $C_{j}$ and $C_{i}$, and $k$ is the size of the set of edges connected to the concept $C_{j}$. The function $f$ is a threshold function (e.g., $f(x)=\tanh (x)$ ), which squashed the $V_{j}^{t}$ into the interval $[-1,1]$, where the value -1 means the worst rating value for a given concept, 0 means a neutral rating, and 1 means the best value.

Also, as shown in Figure 1, the value of an input node is computed through a set of criteria $\mathbf{C}=\left\{c_{1}, c_{2}, \ldots, c_{n}\right\}$. These criteria are represented by the interval $[-1,1]$, such as the nodes of FCM, and express the satisfaction level of the appraiser agent regarding the role played by the target agent. Therefore, each criterion $c_{i}$ makes part of the evaluation performed by the appraiser agent concerning the behavior of a target agent. Whereas the output node of the FCM is represented by the triple Trust (target, role, value) since the trust degree (value), associated with the target agent, depends on the role played by it.

At last, the values assigned to edges of an FCM belong to the interval $[-1,1]$, where -1 means a negative causal relationship, 0 means neutral, and 1 means a positive relationship (Figure 2 (e)). Thus, when two concepts are connected by a positive edge, increasing the value of one of the concepts 


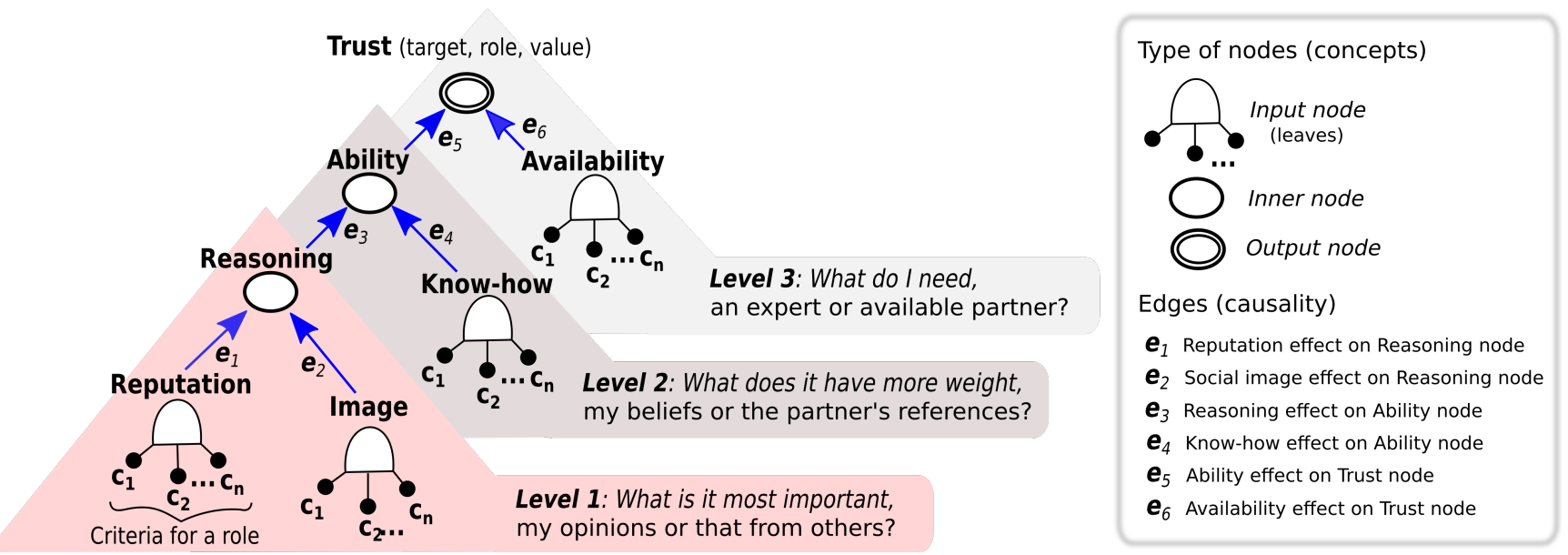

Figure 1. The proposed trust model and its elements. The trust in a target is computed based on a role played by it, considering the $n$ evaluation criteria $(c)$ associated with such a role.

causes a proportional increase in the value of another concept (positive causality). On the other hand, when the concepts are connected by a negative edge, increasing the value of one of the concepts causes a proportional decrease in the value of the other concept (negative causality). Finally, when the causality between two concepts is neutral, changes in the value of one of the concepts do not affect the other.

\subsection{Roles}

In our approach, an appraiser agent may generate a different FCM for each target's skill. In Figure 1, the leaf nodes represent the criteria associated with the evaluated skill of the target agent, and the other nodes represent the essential features associates with the trust.

In this context, we consider that an agent may have several skills, and each of them can be evaluated by different criteria. Thus, an agent may receive distinct ratings about its skills according to the needs and expectations of an appraiser agent. Consequently, an agent can play more than one role in the society and receive distinct evaluations for each one of them. For instance, an agent playing the role of doctor may be evaluated by its treatment administration skill considering criteria like the number of recovered patients, time of recovering, and degree of treatment side effects. At the same time, acting as musician, this agent could be evaluated by its skill to play the guitar.

To estimate the trustworthiness of a target agent, our model considers the social image and reputation of this target as well as its know-how. The know-how of a target represents the previous services provided by it for the other agents (i.e., target's references). The model also considers the target's availability, which indicates whether the target is available to perform the task at a given moment. Moreover, as presented in Figure 1, our model is divided into three levels, wherein at each level, the appraiser agent has to decide what is most important for its evaluation. The levels of the model are discussed in more detail in the following sections.

\subsection{Level 1: Opinions Relevance}

In the first level of the trust model, the appraiser agent calculates the relevance of its own impressions regarding the impressions received from other agents. As discussed in [2], social image and reputation may have different relevance for an agent. In this sense, the personal opinion of an agent may be more important and significant for it than the opinion received from third party agents, and vice-versa.

In particular, for an appraiser agent, the relevance given for the social image or reputation of someone depends on its self-confident profile. Note that in Figure 2 (a) the selfconfident profile of an agent is defined as a fuzzy variable, where the linguistic term low means the agent only believes in what has been said by third parties (reputation), the term middle indicates that the same relevance is given for both the social image and reputation, and finally, the term high means the agent only believes in its own impressions (social image).

\subsection{Level 2: Number of Impressions}

In the second level of our model, the appraiser agent must decide whether the references of a target agent are more important than its own opinion about this target. Thus, the appraiser agent must take into account the number of impressions used to compute the social image $($ imp own $)$ and reputation ( $i m p_{\text {others }}$ ) of the target, as well as, the number of references provided by the target (i.e., the target's know-how) $\left(i m p_{r e f}\right)$. In general, the impressions used in computing the social image, reputation and references of agents are mapped to fuzzy linguistic terms, as shown in Figure 2 (b), 2 (c), and 2 (d), respectively. Note that the linguistic term none represents a situation where the agents have not interacted with others, which usually happens at the beginning of system execution. Therefore, when the system is started, the agents receive a high value of trust. Thus, all agents have at least one chance to interact with an appraiser agent.

On the other hand, as the number of impressions grows, as greater is the effect that the opinions of the appraiser agent 
and references of the target agent have on the estimation of the target abilities. However, as pointed [9], it is important to remark that the reliability degree of the social image, reputation, and references increases until it reaches a maximum value called the intimate level of interactions (ITM). Beyond this ceil more experiences will not increase the reliability of agents' opinions and their references. In general, the value defined for ITM depends on the number of agents and the number of interactions performed by them.

\subsection{Level 3: Urgency}

In the third level of our trust model, the appraiser agent must evaluate how important the availability of a target is for it. This decision is associated with the context and the needs of the appraiser agent. For instance, as presented in [4], in an emergency medical scenario, wherein there is a need to identify what is happening as soon as possible (e.g., in a scenario of heart attack), the urgency of the situation may force the agent to select the first doctor who finds instead to wait for an expert doctor. This example illustrates a situation where the service's urgency is more important than the target's skills. As presented in Figure 2) (a), the urgency is modeled as a fuzzy variable, where the value -1 means the target's availability is more important than its abilities, 0 means they are equally important, and 1 means the abilities of the target are more important than its availability.

\subsection{Fuzzy Classifier}

As previously discussed, the value assigned to an edge that connects a concept $C_{i}$ to a concept $C_{j}$ depends on the importance of these concepts for the agent. In turn, the importance of a concept is defined based on the context of the agents. Herein, we consider that the context is determined by the fuzzy variables urgency, self-confidence, $i m p_{\text {ref }}$, imp $p_{\text {others }}$ and $i m p_{\text {own }}$, as shown in Figure 2 (a), 2 (b), 2 (c), and 2 (d), respectively. For example, if the value high is assigned to the variable urgency, it means an emergency scenario, where finding a partner able to execute a given task is more important than waiting for an expert partner. In this case, the importance of the Availability concept (Figure 1) is higher than the importance of the Ability concept. This situation is represented by rule 3 in Table 1.

In this sense, note that the trust measure estimated by the model tend to change automatically according with the context of agents (i.e., based on the causal relationships defined by the edges of the trust model). This process is controlled by the fuzzy classifier, which operates through a static fuzzy rule base that represents all the possible scenarios of assignment of importance for the concepts of the FCM. In this work, the fuzzy rule base was built manually since the agents cannot learn new rules as they interact with each other.

It is important to remark that the addition of new concepts to the trust model requires the building of new fuzzy rules. In particular, the number of new rules depend on the number of added concepts, the number of edges used to connect these

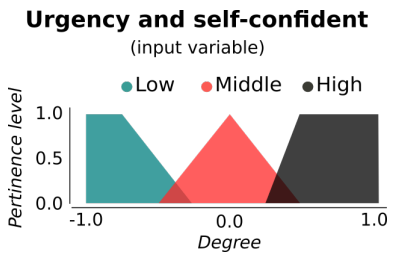

(a)

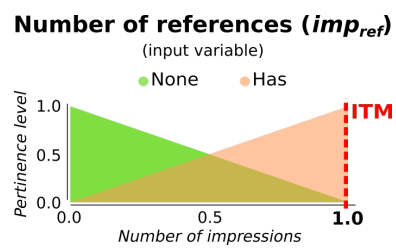

(b)
Number of third-party impressions (impothers) (input variable)

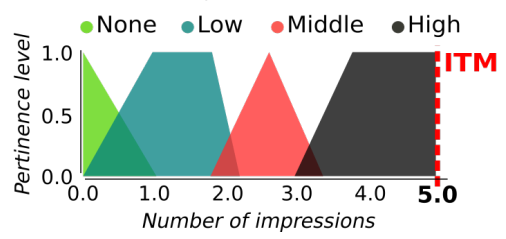

(c)

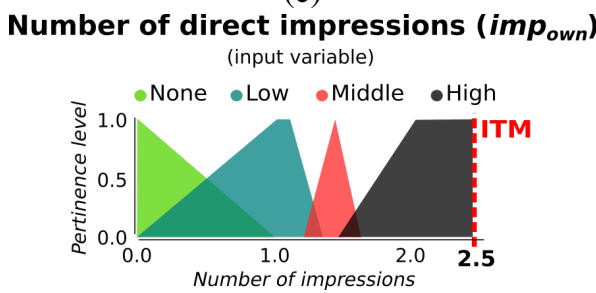

(d)

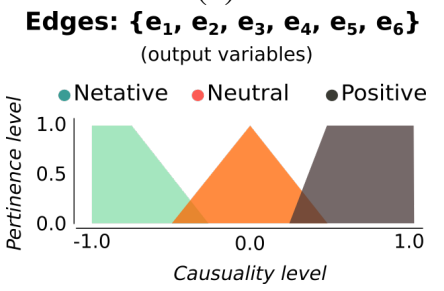

(e)

Figure 2. Input and output variables of the fuzzy classifier: (a) fuzzy values for urgency and self-confidence variables, (b) fuzzy values for $i m p_{\text {ref }}$ variable, (c) fuzzy values for imp others variable, (d) fuzzy values for imp $p_{\text {own }}$ variable, and (e) fuzzy values for the edges of model.

concepts, and the linguistic terms associated with each fuzzy variable.

\section{Commercial Transaction System}

In this section, we present the details about our case study, which implements a commercial transaction system, where products are bought and sold by the agents through commercial transactions (i.e., a contract that establishes the terms and conditions of a transaction). This particular case study was extracted from [9].

\subsection{The scenario}

In the commercial transaction system there are two distinct groups of agents, Buyers, represented by set $\mathbf{B}=\left\{b_{1}, b_{2}, \ldots, b_{n}\right\}$ and Sellers, represented by set $\mathbf{S}=\left\{s_{1}, s_{2}, \ldots, s_{m}\right\}$. Buyer agents send requests for acquiring some products from the 


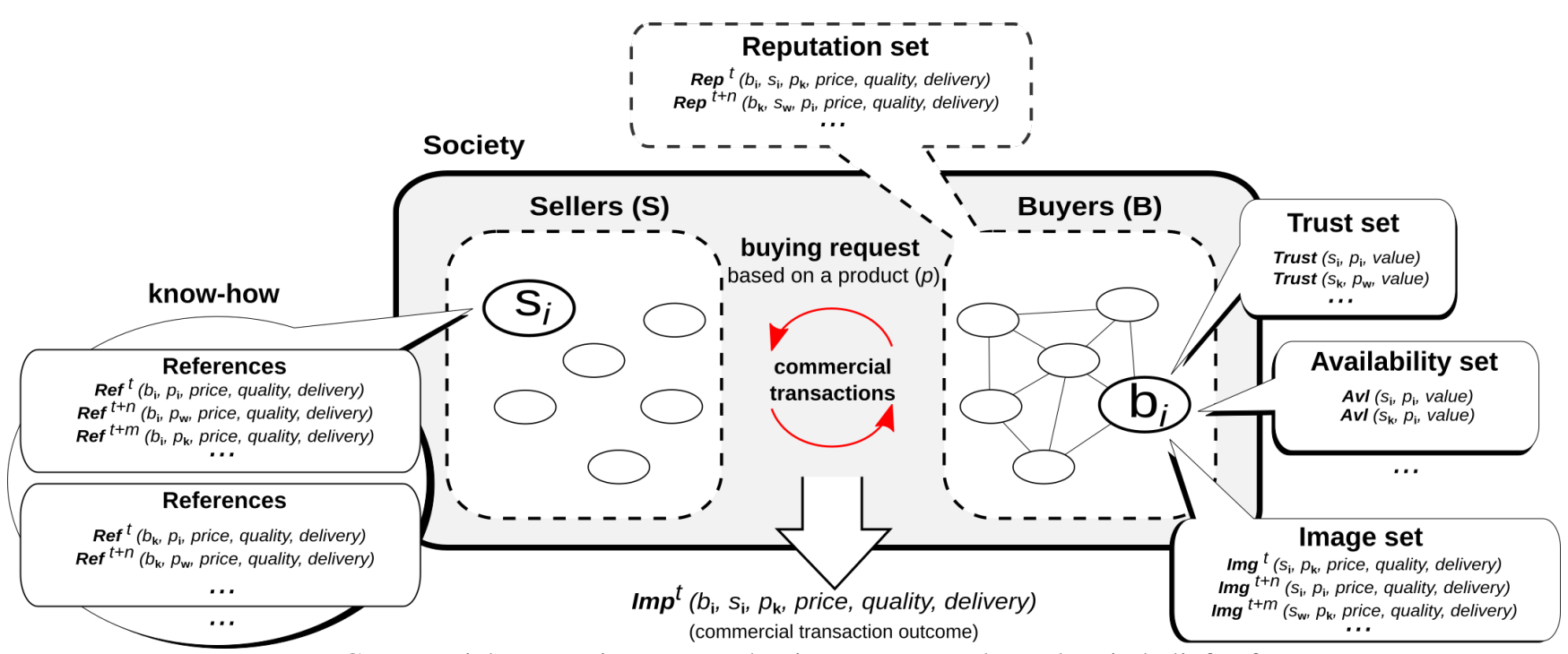

Figure 3. Commercial transaction system, buying request cycle, and main beliefs of agents.

seller agents. Each buying request results in a commercial transaction that represents a contract between a seller and a buyer. When a commercial transaction ends, the buyer generates an impression about the seller's behavior, as Figure 3. This impression is stored by the buyer as a direct experience and used to compute the social image about the seller. As all buyers are connected, every time a buyer produces a new impression, it shares this impression with other buyers (i.e. the buyer contributes to the creation of the seller's reputation). Furthermore, the impression produced by the buyer is also sent to the seller. As presented in Figure 3, this impression is stored by the seller as a service reference, which specifies the buyer who requested the service, the product purchased by the buyer, and the outcome of evaluation made by the buyer about the seller's behavior (i.e., the commitment of seller to keep the original contract conditions concerning price, quality and delivery time of the product). Thus the seller can add such an impression to its know-how and share it with other buyers in future commercials transactions as part of its references. In conclusion, at the beginning of each commercial transaction, the buyers use the trust model to select the most trustworthy seller among all candidates based on information about social image, reputation, references, and availability of sellers.

\subsection{Ratings}

As defined in [9] and [10], a commercial transaction can be seen as an agreement between two agents that specify the terms and conditions of a commercial contract (e.g., the expected quality and the delivery date of a certain product purchased by someone). Thus, when an agent $X$ buys a product from an agent $Y$ through a commercial transaction, an agreement is done, which represents the contract conditions agreed by both agents. In our case study, a contract is defined in terms of price, quality, and delivery time of a product $p$.

For example, the contract $C_{X Y}$ could be the result of a commercial transaction between agent $X$ and agent $Y$, where
$C_{X Y}:=\left(p_{\text {price }}==500 \wedge p_{\text {quality }}==85 \% \wedge p_{\text {delivery }}<=\right.$ 15). It means that agent $X$ expects paying 500 dollars for a given product $p$ with a quality of $85 \%$. Such product must be delivered within 15 days by agent $Y$. When $C_{X Y}$ is established, an expectation is created by agent $X$ in relation to agent $Y$. As discussed in [14], agent $X$ believes that agent $Y$ will commit to the terms of the contract. Thus, when the product is delivered by agent $Y$, agent $X$, based on its expectations, can evaluate agent $Y$. In order to make such an evaluation, agent $X$ must consider its satisfaction concerning the service provided by agent $Y$.

As a result of the evaluation, the agent $X$ is going to produce a rating $r^{t}:=(X, Y, c, v)$ for each contract term (price, quality, or delivery time), where $X$ represents the appraiser agent, $Y$ the target agent, $t$ the time in which the rating was produced, $c$ the evaluated contract term, and $v$ the value associated with the contract term, which belongs to the interval [0, 1 ], where 0 means a bad rating, 1 means an excellent rating, and 0.5 means neutral rating.

Therefore, the impression $\operatorname{Im} p^{t}:=\left(b_{i}, s_{j}, p_{k}, v_{1}, v_{2}, v_{3}\right)$ is produced from the ratings $r^{t}$ performed by a buyer $b_{i}$, at the time $t$, about the behavior of a seller $s_{j}$ with respect to the delivery of a product $p_{k}$, where $v_{1}, v_{2}, v_{3}$ are the values $(v)$ associated with each evaluated contract term $(c)$, price, quality, and delivery time, respectively. It is important to remark that ratings about a seller may become more and more negative according to its lack of commitment with which have been promised.

\subsection{Buyer Agents}

Buyers start the commercial transactions asking for a product. Each buyer $b \in \mathbf{B}$ has its own list of wishes, which consists of products that may or not be acquired as a result of a commercial transaction. In some cases, when there are no sellers who sell the product specified by the buyer, the current transaction is ended and defined as canceled. Additionally, a transac- 
tion may be defined as aborted when there are no trustworthy sellers to sell the wished product. In particular, a buyer only stops making new transactions when its list of wishes is empty. Moreover, each buyer can have its own buying preferences, and a buyer can define which criteria are more relevant to it. For instance, the following relevance tuple $p_{b_{i}}:=$ (price $=1$, quality $=0$, delivery $=0.5$ ), indicates that the buying preferences for the buyer $b_{i}$ assigns the highest priority for low price, the product quality is not important, and the delivery time is partially relevant.

As shown in Figure 3, a buyer stores different beliefs in its belief base and shares the reputation of sellers with other buyers in the society. As previously discussed, social image and reputation beliefs are computed through the aggregation of own and third party impressions, respectively. The availability of a seller is computed based on the number of times that it failed to deliver a product (i.e., the seller has no longer the product in stock). Finally, the trust in each seller is updated every time the seller performs a transaction. Besides, as all beliefs contain a reference to the product sold by the seller, a buyer may have different opinions about a seller according with the product sold by it. For instance, for a certain buyer, a seller may be seen as very trustworthy when selling a product $p_{i}$, and, at the same time, it can see as an untrustworthy seller when selling a product $p_{j}$.

\subsection{Seller Agents}

Each seller $s \in \mathbf{S}$ has its own list of products for sale, which may or not be exclusive. The main goal of a seller is to try to satisfy the buying requests. Nevertheless, more than one seller can answer a given buying request. In this case, a race condition is established, which is solved by the buyer by considering its buying preferences or ranking the sellers based on their trustworthiness. Besides, the sellers may make mistakes, proposing a contract that they will not be able to fulfill. The severity degree of the mistake made by the seller depends on the sale pattern associated with the product sold by it. In particular, the severity degree belongs to the interval $[0$, 1], where 0 means no mistake, and 1 means that the product delivered by the seller is totally out of the specifications denied in the contract. The sales patterns are discussed in detail in Section 5. As shown in Figure 3, sellers may stores beliefs regarding their past sales in order to produce their references (know-how). These references may be shared with the buyers when the seller sends a proposal to them.

\subsection{Social Interactions}

In order to ensure the correct functioning of commercial transactions, the contract net protocol (CNP) was implemented. The CNP was proposed by [21] and is used to allocate tasks among autonomous agents. The protocol starts when a buyer $b \in \mathbf{B}$ receives a perception from the environment about the product that must be purchased. At this moment, a call-forproposal $(c f p)$ is sent to all sellers. In this call, the buyer $b_{i}$ specifies the product that wants to buy and the identifier of call (id), since several $c f p$ may be triggered at the same time by different buyers. When a seller $s \in \mathbf{S}$ receives a $c f p$ and has the requested product, such a seller sends a proposal to the respective buyer. The sent proposal contains the contract conditions defined by the seller, which may be or not fulfilled in the future. In turn, upon receiving all proposals, the buyer $b$ analyzes each one and selects the best one based on its buying preferences (e.g., low-price, high-quality, and short delivery time) and the sellers' trustworthiness. Finally, after the seller delivers the product, based on its expectations, the buyer evaluates the seller and produces its impression. Such an impression is shared with the other buyers, as part of the seller's reputation, as well as, with the seller, as part of the seller's references.

\section{Experiments and Results}

In this section, we present the results of our experimental study. All of the experiments presented herein are performed considering scenarios where buyer agents interact with seller agents to buy some products. The buying requests happen concurrently, and hence, the buyers compete among them for the best sellers. However, each buyer can buy a product at a time. On the other hand, a seller can negotiate with several buyers simultaneously, selling its products in stock. When the stock is empty, the seller spends one interaction to restore the stock.

\subsection{Experimental Setup}

In our experiments, all agents are designed through BDI architecture [22]. Thus, the relationships defined among agents are represented in terms of cognitive elements such as beliefs, desires, and intentions. For example, motivated by its desire to purchase a product, a buyer agent, based on its beliefs, may judge a seller agent as trustworthy and send a buying request to it to obtain the desired product. (i.e., executing a plan to reach its goal of purchasing a product). In particular, all agents were implemented in Jason [23], an interpreter for an extended version of AgentSpeak [24].

As each buyer agent has an instance of the trust model, some configurations must be performed to ensure the correct model operation. Therefore, as the number of impressions shared by the agents grows faster than the number of direct impressions produced by them, we consider the ITM value associated with the social image is twice lower than the value assigned to the reputation. In turn, the reliability degree of the know-how of an agent reaches its maximum value when at least one reference is produced (ITM equals 1). An example of these constraints can be observed in Figure 2 (b), 2 (c), and 2 (d). Note that the maximum reputation level is reached when the ITM value is 5 (Figure 2 (c)), for the social image, the maximum level is reached when the ITM is 2.5 (Figure 2 (d)), and for the know-how level, when ITM is greater than 1 (Figure 2 (b)).

Another important configuration is the method employed to build the fuzzy rule base used by the fuzzy classifier. For the experiments presented herein, the fuzzy rule base is created 


\section{Antecedent}

\section{Rule 1: IF urgency low}

Rule 2: IF urgency middle

Rule 3: IF urgency high

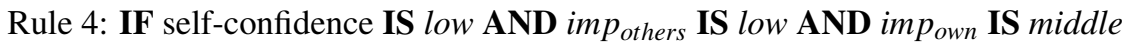

\section{Consequent}

THEN $e_{5}$ positive AND $e_{6}$ neutral

THEN $e_{5}$ positive AND $e_{6}$ positive

THEN $e_{5}$ neutral AND $e_{6}$ positive

THEN $e_{1}$ positive AND $e_{2}$ neutral

Table 1. Fuzzy Rules used to define the values of edges of the trust model considering the input variables self-confidence, $i m p_{\text {own }}$, imp $p_{\text {others }}$, and urgency. Edges: $e_{1}$ (Reputation effect on Reasoning concept), $e_{2}$ (Social image effect on Reasoning concept), $e_{5}$ (Ability effect on Trust concept), and $e_{6}$ (Availability effect on Trust concept).

manually without an automatic generation method. In such a rule base, there are 167 rules, being 96 of these rules produced based on the self-confident, $i m p_{\text {own }}$, and imp $p_{\text {others }}$ variables. Such rules control the edges $e_{1}$ and $e_{2}$ in the first level of the trust model. On the other hand, based on imp $p_{\text {ref }}$, imp $p_{\text {own }}$, and imp $p_{\text {others }}$ variables, other 65 rules were created to control the edges $e_{3}$ and $e_{4}$ in the second level of the model. Finally, the last 6 rules control the edges $e_{5}$ and $e_{6}$ in the third level of the model. Note that only the urgency variable is associated with these rules. In particular, the fuzzy classifier was implemented in Java through jFuzzyLogic library [25].

In order to exemplify the use of the fuzzy rules, in Table 1 some rules are shown. In Rule 1, when the value low is assigned to the urgency variable, a positive causal relation is defined between the Trust and Ability concepts (edge $e_{5}$ of the FCM shown in Figure 1), simultaneously a neutral causal relation is defined between the Trust and Availability concepts (edge $e_{6}$ ). In this case, when the value of the Ability concept is changed, such a variation is directly propagated to the Trust concept. On the other hand, the effects of the Availability concept on the Trust concept are canceled. A similar situation is shown in Rule 4, where the self-confidence variable determines the relevance of Social image and Reputation concepts concerning Reasoning concept. Note that in this situation, as the value low is assigned to the self-confidence variable, the relevance given for the Reputation concept is higher than the Social image concept (edge $e_{1}$ ). In this case, the effect of the Social image on the Reasoning concept is canceled due to the neutral causality established by edge $e_{2}$.

\subsection{Sale Patterns}

In our experiments, we are using behavioral functions to simulate different social behaviors. These functions are applied to products sold by the sellers. Thus a seller can assume different behaviors according to the product sold by it. A behavioral function defines the severity degree of the mistake made by a seller to estimate the conditions of a contract. The higher the severity degree, the higher is the chance of the seller do not get to fulfill the terms of a contract. In this situation, the buyer's expectations concerning the product sold by the seller are not meet, which decreases the buyer's trust in the seller. In general, the severity of the mistakes made by a seller may change over time according with the type of behavioral function adopted. The behavioral functions considered in our experiments are described in the following:
- Constant-function: this function is used to represent expert sellers. The sellers that sell products associated with this kind of function do not make mistakes. Therefore, such sellers deliver their products to buyers keeping the conditions promised in the contract.

- Linear-function: this function represents a situation where a seller starts a business very well but, over time, it loses its credibility due to its estimation mistakes. In this case, the sellers can overestimate or underestimate the value of one or more contract terms before delivering the product to a buyer. The equation adopted to simulate this behavior is shown in Equation 3. When a seller assumes this behavior, the evaluations received by it about its behavior become more and more negative, resulting in the decay of its trustworthiness.

- Exponential-function: this function is very similar to the Linear-function, except for its decay rate, which is more smooth, being represented by an exponential curve. The equation adopted to simulate this behavior is shown in Equation 4.

Concerning Equation 3 and Equation 4, $n$ represents the number of sales necessary for a seller to double the severity degree of its mistakes. For example, assuming $n$ equals two, and the delivery time as the term of the contract to be underestimated, after its second sale, the seller will double the number of days needed to deliver a product. Thus, even the seller has estimated two days to make the delivery, it is going to take four days to deliver the product. In turn, $x$ represents the number of sales already made by a seller. In particular, $x \in[0, n]$, where 0 means no sale made, and $n$ means the number of sales needed to double the severity degree of the mistakes made by a seller.

$$
\begin{aligned}
& f_{L}(x)=1-\frac{x}{n} \\
& f_{E X P}(x)=1-\frac{e^{x+\frac{n}{3}}}{e^{n}}
\end{aligned}
$$

\subsection{Evaluating the Agents' Options}

The goal of this experiment is to evaluate the social image and reputation effects on trust computation. As presented in Figure 1, the social image and reputation are the inputs for the Reasoning concept, which defines how relevant the own 
experiences of an agent are in regards to what is being said about a target in the society. The relevance given for the social image or reputation may change according to the agent's context. For instance, when an agent has few connections (i.e., the agent is new or an isolated agent), it may decide to trust only in its own experiences. On the other hand, as an agent interacts with others, sending and receiving evaluations about the behaviors of other agents, such an agent may decide to trust as well in the evaluations received from third parties.

To evaluate the relationship between social image and reputation, we perform three different experiments. In the first, the buyers evaluate the sellers just using their social image about each seller. In the second experiment, the buyers only consider the sellers' reputation. In the last, the social image and reputation are defined as equally relevant for the buyers. For these experiments, in our trust model, the edge that connects the Availability concept to the Trust concept is defined as neutral. Thus, the buyers can select sellers based on just the sellers' abilities.

Each experiment is composed of 5 buyers and 3 sellers. Moreover, each buyer has a list of wishes for 20 products, all of them of the same type (product $A$ ), which are purchased in a specific sequence. Concerning the buying preferences, two buyers search for low-price and high-quality products, two buyers search for high-quality products and short delivery time, and for the last buyer, all buying preferences are equally important (i.e., low-price, high-quality and short delivery time). On the other hand, to create competition condition among the sellers, they sell the same products, product $A$, and they always have products in stock. Besides, each seller has its own behavior, where the Constant-function is associated with the products sold by seller 1, the Linear-function is associated with the products sold by seller 2, and the Exponentialfunction is associated with the products sold by seller 3 .

The average of trust computed by buyers along the interactions with sellers is shown in Figure 4. In Figure 4 (a), the trust values are computed based only on the social image. In Figure 4 (b), the trust values are computed only considering the reputation of the sellers. In Figure 4 (c), the same relevance is given for social image and reputation. Notice that when just the social image is adopted, the trust in the sellers 2 and 3 tend to decrease quickly, being below the trust threshold (0.2) at iteration 9. On the other hand, where just reputation is considered, the trust in the sellers 2 and 3 tends to decrease slower than the social image approach. Moreover, as presented in Figure 4 (b), the trust in sellers 2 and 3 is always above the trust threshold. In particular, the trust variation presented in Figure 4 (a) and Figure 4 (b) can be associated with the number of impressions that are produced for computing the social image and reputation. As discussed in [3] and [2], the social image tends to be more sensitive to the number of impressions produced by agents than reputation, since the number of direct impressions produced by an agent is much smaller than the number of impressions received from third parties. Finally, in Figure 4 (c), the sellers 2 and 3 interact for a long time with buyers before they become untrustworthy. Besides, due to the difference between the number of impressions used to compute the social image and reputation of the sellers, when compared to Figure 4 (a) and Figure 4 (b), in Figure 4 (c), the effects from the social image and reputation on the trust values produced by the model are balanced.
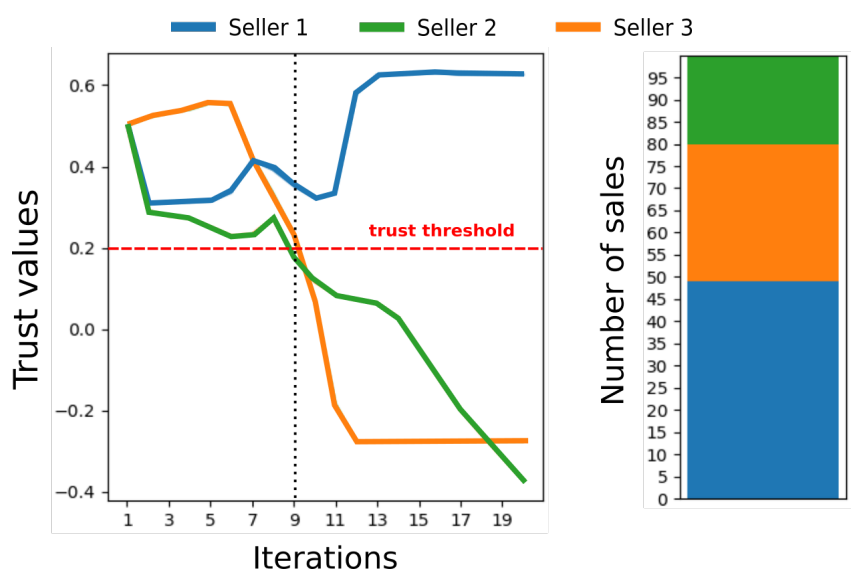

(a)
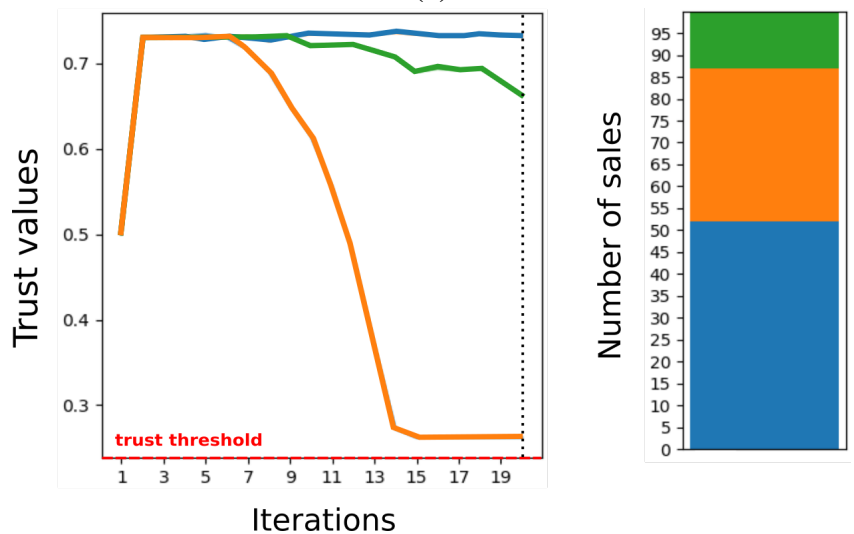

(b)
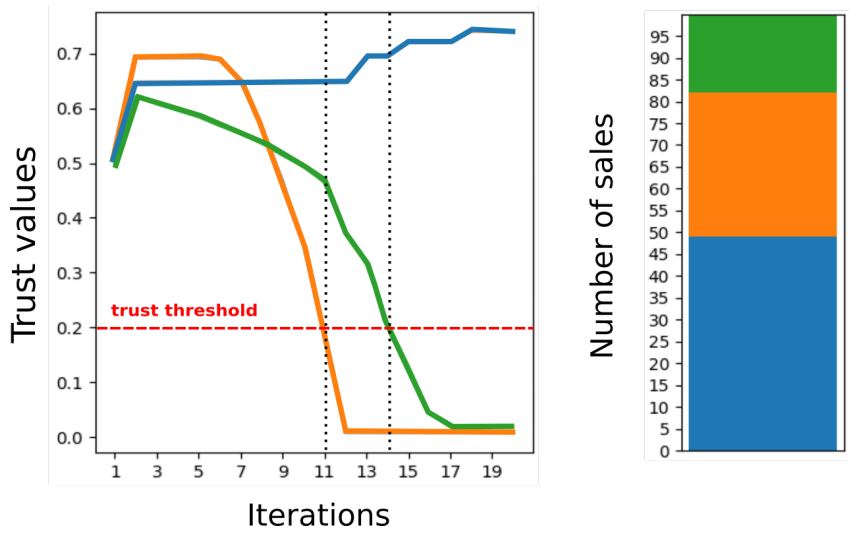

(c)

Figure 4. Average of the buyers' trust in the sellers: (a) trust average computed only using social image information, (b) trust average computed only using reputation information, and (c) trust average computed using the social image and reputation information. 


\subsection{Evaluating Ability and Availability}

In this experiment, we discuss how our model can be configured to deal with the partner selection process. In general, several trust and reputation models in literature make use of the abilities and expertise of agents to select good partners [1] [26] [7]. Nevertheless, as discussed in [4], for some applications, the best partner may not be the most skilled agent. For instance, evaluating the possible partners considering the urgency of a task may be interesting in a situation where there is a high competition for the services provided by expert agents (i.e., waiting a long time for an expert agent might be not feasible). Therefore, an agent should be able to select its partners using the evaluation criteria most appropriate to its context.

This experiment is composed of 2 buyers, (Buyer 1 and Buyer 2), and one seller. Each buyer has a list of wishes of 20 products, which are divided into products of type $A$ and $B$. Moreover, these products are purchased in a specific sequence, where for each product of type $A$, other 4 products of type $B$ are purchased. Concerning buying preferences, both buyers search for products with low-price, high-quality, and short delivery time. We assume the Buyer 1 represents an agent concerned with the availability of the seller, and thus, its urgency level is defined as 0.7 (i.e., this buyer prefers sellers with high availability and does not worry about their skills). In turn, the Buyer 2 searches for expert sellers, and its urgency level is defined as -0.3 (i.e., this buyer prefers high-skilled sellers does not worry about their availability). In turn, the seller sells products of type $A$ and $B$, but the stock of each product is limited to 2 units. Thus, for every two units sold, the seller must replenish its stock. Finally, the Constant-function is associated with each product sold by the seller.

As presented in Figure 5, the seller's availability decreases for every two units of product sold. In particular, when the seller tries to sell a product that he doesn't have in stock, the sale is canceled, and its availability level decreases. In this way, in Figure 5 (a), the trust that the Buyer 1 has in the seller tends to decrease over time since such a trust depends on the seller's availability. Thus, for the Buyer 1, the seller becomes an untrustworthy agent as time goes. On the other hand, in Figure 5 (b), the seller's availability does not significantly affect the trust of the Buyer 2, because the evaluations performed by the buyer are based on the seller's abilities (i. e., the social image, reputation, and know-how). Thus, as the seller delivers its products as promised, for the Buyer 2, the seller is seen as a trustworthy agent.

\subsection{Results Analysis}

In our experiments, we remark that the social image takes more effort from agents to be produced than reputation information. To create a social image about another agent, the agents need to interact directly with it. As pointed in [3], every time an agent interacts with an unknown target, such an agent is putting itself in a risk position since this target may be a cheater agent.

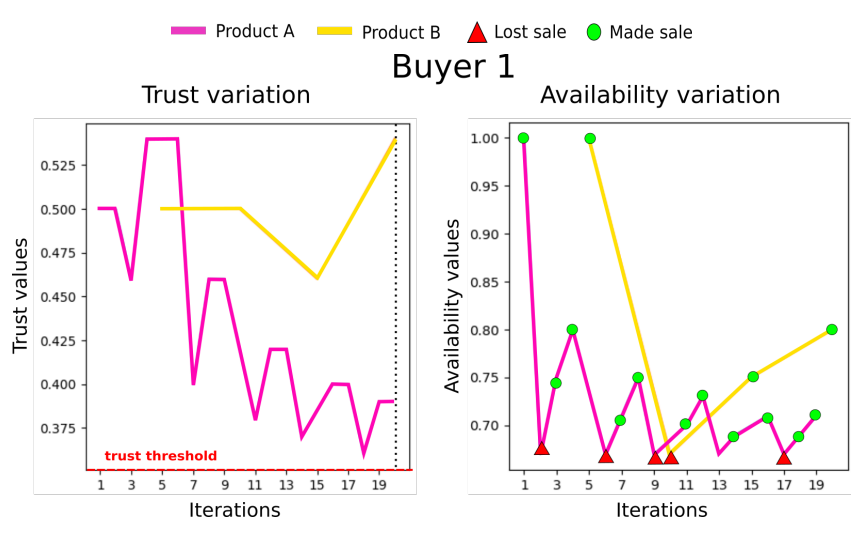

(a)

Buyer 2
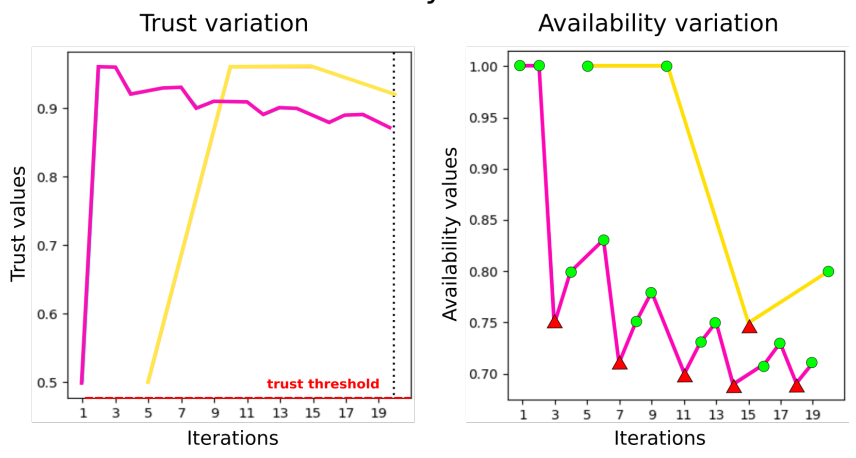

(b)

Figure 5. Trust and availability variation considering the interactions among the buyers and the seller: (a) trust and availability computed by Buyer 1, and (b) trust and availability computed by Buyer 2 .

Concerning reputation, the agents may judge a target as good or bad even they have never interacted with it before because such an assessment is performed based on the third party impressions shared with them [2]. However, as discussed [10] [27] [16], in a situation where the agents can share fake information with others, the use of reputation may be a problem. In this situation, the social image can be used as an efficient mechanism to deal with fake information. As pointed in [2], the social image tends to be a trustworthy source of information since it is produced based on the direct experiences of agents. For example, when the social image and the reputation of a target are in agreement, there is a good chance that the target's reputation may be true. In this case, the direct experiences of the agent support what is said in society about the target's behavior.

In particular, in our trust model, when the social image and reputation are opposite (uncertain situation), the value assigned to the Reasoning concept (in the FCM) becomes neutral, decreasing the trust value produced by the model. Furthermore, from a cautious point of view, our trust model can be configured to give more relevance to the Social image than other concepts, as reputation and know-how. This configuration allows the agents to trust in their own experiences 
instead of the experiences of other agents. Another important aspect of our trust model is that it can be easily configured to deal with different evaluation criteria. For instance, as presented in our experiments, the buyers can decide to evaluate the sellers by ability or availability. Such flexibility can be explored in open MASs [28]. Since in this type of application, the agents can join and leave freely into the system. In this case, new demands may be requested, which might change the evaluation criteria used by agents to select their partners.

\section{Conclusions}

In this paper, we presented a case study where agents interact with each other in a commercial transaction system. In particular, buyer agents perform buying requests for certain products, and seller agents aim to provide the requested product. The sellers are rated (evaluated) after the product delivery by their respective buyers. A rating expresses the seller's reliability regarding the contract terms (i.e., price, quality, and delivery time of the negotiated product). For buyers, the ratings are translated to impressions used to update their trust in the seller.

In our experiments, we have noted that when the social image is adopted, the behavior variations of the agents are easily perceived during trust computing. On the other hand, due to the volume of impressions produced and shared by the agents, the trust values calculated based on reputation tend to be higher than the trust values computed base on the social image. Moreover, we observed that the inconsistencies among the sources of information (e.g., social image, reputation, and references) are naturally solved by the FCM structure, in which the sources of information are combined based on their relevance (degree of importance) for the agents. Another contribution of this work is the dynamism of our trust model. In the approach presented herein, an appraiser agent may generate a different FCM for each role played by a partner. This feature allows agents to evaluate their partners based on the distinct roles played by them, even for situations where the agents are able to learn new roles. Moreover, by using our trust model, an appraiser agent can make decisions about their partners taking into account different sources of information (perspectives). Furthermore, the agents can determine the reliability of a source of information by associating a degree of importance with it. Thus, in dynamic scenarios, where not all the sources of information might be available at the same time, the agents can select the most stables of them to compute the trust measure.

As future work, we intend to investigate ways for making the model configurations fully automatic since the fuzzy rules used by the FCM are built manually. In this sense, the agents could learn about the environment as they interact with each other and dynamically add new rules to their rule base. Another possible investigation is the study of the risks associated with the act of trusting someone. In particular, the risks associated with external factors $(e . g$., the environment condition, dependencies of resources, and goal conflicts). This type of modeling requires a more accurate analysis of the objectives and resource dependencies among the agents.

\section{Related Works}

As previously discussed, trust is a fundamental component to build effective MASs. The adoption of a trust model allows autonomous agents to make decisions about the selection of partners and coordinate with them [29]. According to [7], in the two past decades, a considerable amount of researches has been conducted on the topics of computational trust and reputation models. For instance, in [4], the authors present a $\operatorname{cog}$ nitive trust model built based on an FCM. In this model, the trust assessment is performed through specific beliefs, which represent the essential components of the trust. Whereas in [30], the proposed trust model is focused on handling new agents, for whom the history of interaction is not available. In this case, the authors use stereotypes to evaluate the profiles of new agents. On the other hand, the trust model proposed by [31] aims to evaluate whether an action would be perceived as trustworthy in a context where artificial systems interact with each other. A similar approach is proposed in [32], but in this case, the model is focused on human-robot interaction, where the robots predict the amount of trust that a potential partner (human) has about them. Finally, the trust model proposed in [33] effectively utilize the explicit and implicit influence of trust for rating predictions in the recommendation system.

Concerning reputation models, in the Regret model, the reputation is computed based on a multi-dimension evaluation (i.e., individual, social and ontological dimensions)[9]. In particular, such a model allows agents to have different reputations based on the functions and roles played by them in society. A similar reputation model is proposed in [10], where besides multidimensional evaluation, the authors implement a process of certifying reputation to deal with cheating agents. On the other hand, the Repage model adopts the concept of social image and reputation for identifying trustworthy agents [15]. In this case, an agent is evaluated based on its reputation that circulates in the society, as well as, on the social image that other agents have about it. Additionally, in [2] the Repage model was extended to support BDI agents.

Even though the large amount of trust and reputation models present in the literature, few of them are general-purpose models. Moreover, many of these models are often limited to a particular research domain [13]. In this sense, our trust model can bring great advantageous since it can be adopted in different application domains. Note that independent of application, the basic concepts (inner nodes) of our model remain unchanged. Furthermore, as the impressions produced by agents are based on social image and reputation beliefs, only the criteria used in the leaf nodes must be adjusting according to roles played by agents in a certain situation. 


\section{Acknowledgements}

The authors would like to thank the CAPES (Coordenação de Aperfeiçoamento de Pessoal de Nível Superior), which is fully founded this work.

\section{Author contributions}

All authors have contributed equally to the development of this work.

\section{References}

[1] BUCCAFURRI, F. et al. Experimenting with certified reputation in a competitive multi-agent scenario. IEEE Intelligent Systems, IEEE, v. 31, n. 1, p. 48-55, 2015.

[2] PINYOL, I.; SABATER-MIR, J. Computational trust and reputation models for open multi-agent systems: a review. Artificial Intelligence Review, Springer, v. 40, n. 1, p. 1-25, 2013.

[3] CONTE, R.; PAOLUCCI, M. Reputation in artificial societies: Social beliefs for social order. [S.1.]: Springer Science \& Business Media, 2002. v. 6.

[4] CASTELFRANCHI, C.; FALCONE, R. Trust theory: A socio-cognitive and computational model. [S.1.]: John Wiley \& Sons, 2010. v. 18.

[5] GRIFFITHS, N. Task delegation using experience-based multi-dimensional trust. In: Proceedings of the fourth international joint conference on Autonomous agents and multiagent systems. [S.1.: s.n.], 2005. p. 489-496.

[6] GOVINDAN, K.; MOHAPATRA, P. Trust computations and trust dynamics in mobile adhoc networks: A survey. IEEE Communications Surveys \& Tutorials, IEEE, v. 14, n. 2, p. 279-298, 2011.

[7] BRAGA, D. D. S. et al. Survey on computational trust and reputation models. ACM Computing Surveys (CSUR), ACM New York, NY, USA, v. 51, n. 5, p. 1-40, 2018.

[8] FALCONE, R.; PEZZULO, G.; CASTELFRANCHI, C. A fuzzy approach to a belief-based trust computation. In: SPRINGER. Workshop on Deception, Fraud and Trust in Agent Societies. [S.1.], 2002. p. 73-86.

[9] SABATER, J.; SIERRA, C. Regret: reputation in gregarious societies. In: Proceedings of the fifth international conference on Autonomous agents. [S.1.: s.n.], 2001. p. 194-195.

[10] HUYNH, T. D.; JENNINGS, N. R.; SHADBOLT, N. Fire: An integrated trust and reputation model for open multi-agent systems. 2004.

[11] PINYOL, I. et al. Reputation-based decisions for logic-based cognitive agents. Autonomous Agents and Multi-Agent Systems, Springer, v. 24, n. 1, p. 175-216, 2012.
[12] SOLHAUG, B.; ELGESEM, D.; STOLEN, K. Why trust is not proportional to risk. In: IEEE. The Second International Conference on Availability, Reliability and Security (ARES'07). [S.1.], 2007. p. 11-18.

[13] CHO, J.-H.; CHAN, K.; ADALI, S. A survey on trust modeling. ACM Computing Surveys (CSUR), ACM New York, NY, USA, v. 48, n. 2, p. 1-40, 2015.

[14] CASTELFRANCHI, C.; GUERINI, M. Is it a promise or a threat? Pragmatics \& Cognition, John Benjamins, v. 15, n. 2, p. 277-311, 2007.

[15] SABATER, J.; PAOLUCCI, M.; CONTE, R. Repage: Reputation and image among limited autonomous partners. Journal of artificial societies and social simulation, v. 9, n. 2, 2006.

[16] MAHMOOD, S. et al. Reputation-based approach toward web content credibility analysis. IEEE Access, IEEE, v. 7, p. 139957-139969, 2019.

[17] MUÑOZ, V.; MURILLO, J. Agent uno: Winner in the 2nd spanish art competition. Inteligencia Artificial. Revista Iberoamericana de Inteligencia Artificial, Asociación Española para la Inteligencia Artificial, v. 12, n. 39, p. 19-27, 2008.

[18] BOTELHO, V. et al. Dossier: decentralized trust model towards a decentralized demand. In: IEEE. 2018 IEEE 22nd International Conference on Computer Supported Cooperative Work in Design ((CSCWD)). [S.1.], 2018. p. 371-376.

[19] KOSKO, B. et al. Fuzzy cognitive maps. International journal of man-machine studies, v. 24, n. 1, p. 65-75, 1986.

[20] STYLIOS, C. D.; GROUMPOS, P. P. Modeling complex systems using fuzzy cognitive maps. IEEE Transactions on Systems, Man, and Cybernetics-Part A: Systems and Humans, IEEE, v. 34, n. 1, p. 155-162, 2004.

[21] SMITH, R. G. The contract net protocol: High-level communication and control in a distributed problem solver. IEEE Transactions on computers, IEEE, n. 12, p. 1104-1113, 1980.

[22] RAO, A. S.; GEORGEFF, M. P. et al. Bdi agents: from theory to practice. In: Icmas. [S.1.: s.n.], 1995. v. 95, p. 312-319.

[23] BORDINI, R. H.; HÜBNER, J. F. Bdi agent programming in agentspeak using jason. In: SPRINGER. International Workshop on Computational Logic in Multi-Agent Systems. [S.1.], 2005. p. 143-164.

[24] RAO, A. S. Agentspeak (1): Bdi agents speak out in a logical computable language. In: SPRINGER. European workshop on modelling autonomous agents in a multi-agent world. [S.1.], 1996. p. 42-55.

[25] JFUZZYLOGIC. 〈http://jfuzzylogic.sourceforge.net/ html/index.html $\rangle$. Accessed: 2020-10-29. 
[26] BORGES, A. P. et al. Safety in multi-agent systems: Reputation based on dossier. In: The Twenty-Eighth International Flairs Conference. [S.1.: s.n.], 2015.

[27] SUN, Y.; LIU, Y. Security of online reputation systems: The evolution of attacks and defenses. IEEE Signal Processing Magazine, IEEE, v. 29, n. 2, p. 87-97, 2012.

[28] HENDRICKX, J. M.; MARTIN, S. Open multi-agent systems: Gossiping with random arrivals and departures. In: IEEE. 2017 IEEE 56th Annual Conference on Decision and Control (CDC). [S.1.], 2017. p. 763-768.

[29] ZHANG, J. et al. Introduction to the Special Section on Trust and AI. [S.1.]: ACM New York, NY, USA, 2019.

[30] LIU, X.; DATTA, A.; RZADCA, K. Trust beyond reputation: A computational trust model based on stereotypes. Electronic Commerce Research and Applications, Elsevier, v. 12, n. 1, p. 24-39, 2013.

[31] HAMMER, S.; WISSNER, M.; ANDRÉ, E. Trust-based decision-making for smart and adaptive environments. User Modeling and User-Adapted Interaction, Springer, v. 25, n. 3, p. 267-293, 2015.

[32] LEE, J. J. et al. Computationally modeling interpersonal trust. Frontiers in psychology, Frontiers, v. 4, p. 893, 2013.

[33] HU, Z. et al. Ssl-svd: Semi-supervised learning-based sparse trust recommendation. ACM Transactions on Internet Technology (TOIT), ACM New York, NY, USA, v. 20, n. 1, p. 1-20, 2020. 